\title{
NLC LINAC FEEDBACK WITH GROUND MOTION*
}

\author{
Linda Hendrickson, Thomas Himel, Nan Phinney, Tor Raubenheimer, \\ Andrei Seryi, Peter Tenenbaum, Mark Woodley, SLAC, Stanford, USA
}

\section{Abstract}

Beam-based feedback systems play an essential role in the operation of high energy electron-positron linear colliders. Ground motion, vibration and other disturbances can significantly alter the beam trajectory or degrade the beam quality. Feedback systems are required for long term stability and for ease and efficiency of operation. Trajectory feedback for the Next Linear Collider (NLC) [1] has been studied extensively to understand both the achievable orbit quality and the time response characteristics. More recently, these studies have been extended to evaluate performance in the presence of ground motion.

\section{GROUND MOTION MODELS}

Previous studies of the NLC linac performance with respect to ground motion used the ATL [2,3] model of ground motion, which is only appropriate for simulation time intervals from hours to months. Recently, more realistic ground motion models have been developed [4] that are consistent with all known measurements. In particular they are based on measurements of correlation properties of fast ground motion at CERN, SLAC and DESY and on measurements of slow motion at SLAC, DESY, FNAL and KEK (see references in [4]).

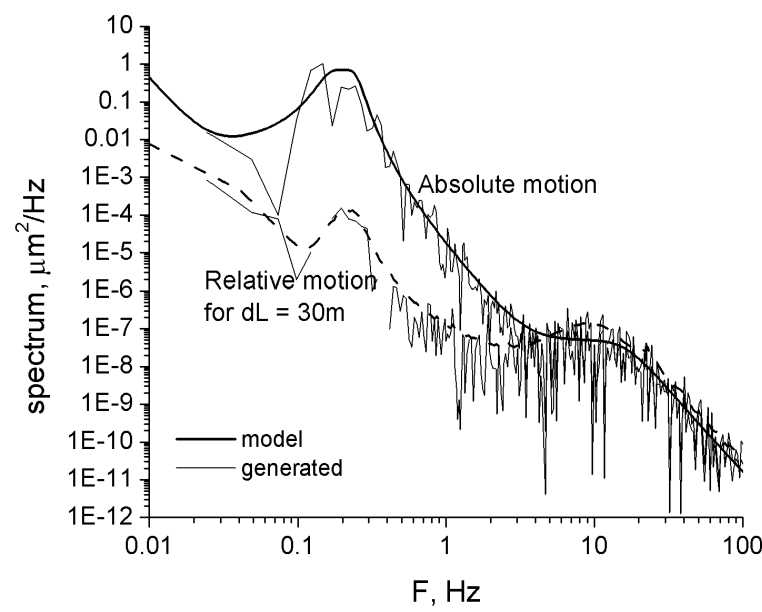

Figure 1: Power spectra of absolute and relative $(\mathrm{dL}=30 \mathrm{~m})$ motion for the 'SLAC model' of ground motion. Smooth lines show the curves used in the model and motion generated and 'measured' within LIAR.
The SLAC model of ground motion [4] used in the simulations presented below corresponds to a shallow tunnel built in sandstone in a moderately populated area. The ground motion model is parameterized by a $2 \mathrm{D}$ power spectrum $\mathrm{P}(\omega, \mathrm{k})$ which is then used to generate transverse misalignments of the linac $\mathrm{x}(\mathrm{t}, \mathrm{s})$ and $\mathrm{y}(\mathrm{t}, \mathrm{s})$. A harmonic summation is used with temporal and spatial frequencies that are distributed equidistantly (with small seed-dependent fluctuations) in the logarithmic sense over an appropriate range of $(\omega, \mathrm{k})[5]$.

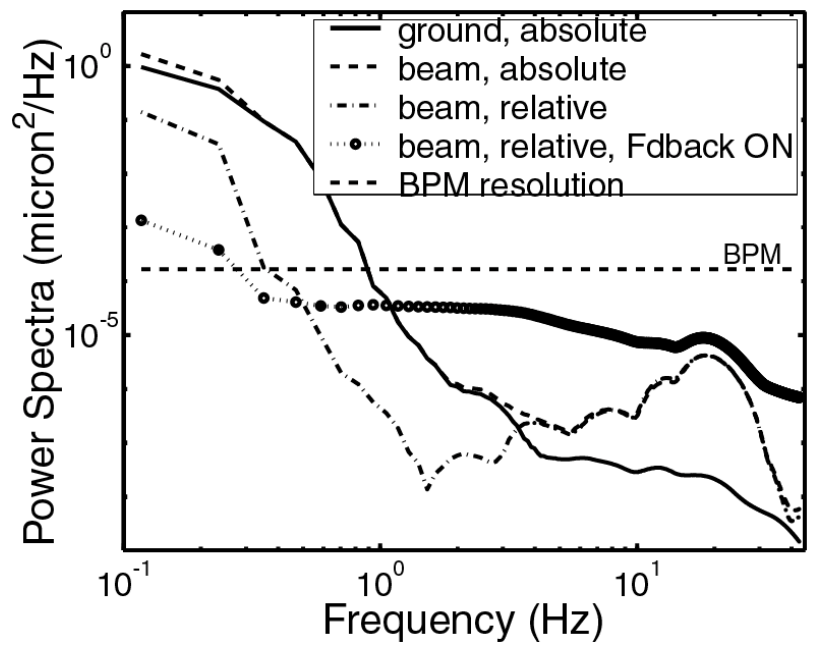

Figure 2: Power spectral density of motion at the end of the linac. Curves show absolute ground motion (with respect to an inertial frame), absolute and relative (with respect to the end of the linac) beam motion without feedback, relative beam motion with feedback orbit correction; spectrum corresponding to BPM resolution 0.1 micron.

This method of ground motion modeling is now included in the LInear Accelerator Research code LIAR [6] - the tool used for these simulations. An example of a ground motion spectrum generated by LIAR is shown in Fig.1. Both absolute and relative spectra of generated motion are consistent with the modeling spectra obtained by direct integration of $\mathrm{P}(\omega, \mathrm{k})$. 


\section{LINAC STABILITY STUDIES}

Feedback orbit correction was developed for SLC $[7,8]$, and was invaluable for stable operation. The feedback decreased operator tuning time, facilitated tuning by decoupling different regions of the machine, and enabled quicker startup after machine outages. Performance limitations of the SLC feedback system have been extensively studied [9] and many improvements are planned for the NLC. The tolerances on orbit stability for the NLC linac are too tight to maintain without feedback. The NLC linac performance has been studied in the presence of ground motion as represented by the SLAC model. Figures $2-4$ show the performance in the high frequency range and Fig.5-6 in the low frequency range.
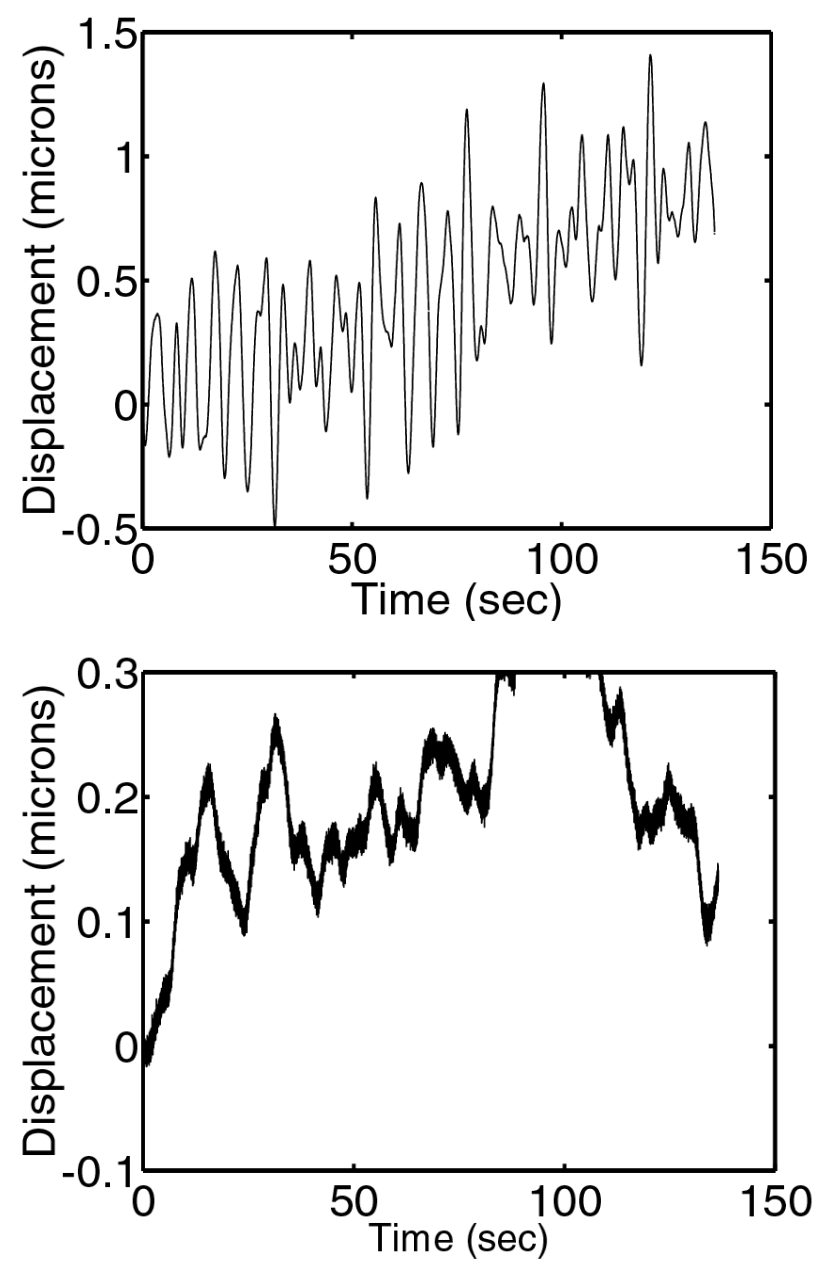

Figure 3: Absolute ground motion at the end of the linac (top) and relative beam motion with respect to the end of linac (bottom) without feedback orbit correction.

Figures 2 and 3 show that without feedback, the beam motion at $\mathrm{f}>4 \mathrm{~Hz}$ is amplified with respect to ground motion. At these frequencies, the ground motion is uncorrelated over the typical distance between quadrupoles. Since many quadrupoles contribute to the beam motion, it is therefore amplified with respect to the ground motion. In contrast, at lower frequencies, the ground motion is very well correlated and the beamline deformation is smooth over one betatron wavelength. Therefore, the absolute beam motion and the ground motion are almost exactly the same at low frequency. The relative beam motion with respect to the linac is correspondingly smaller than the absolute beam motion.

For this simulation, the orbit feedback used BPMs with a resolution of 0.1 micron. It is obvious that since the ground motion is much smaller than this value at high frequencies, the feedback is not effective above approximately $\sim 1 \mathrm{~Hz}$. In fact at high frequencies, feedback increases the motion somewhat. Below $\sim 1 \mathrm{~Hz}$ the beam motion is effectively suppressed by the orbit feedback. Figure 4 shows that the relative beam position at the end of the linac is less than $4 \%$ of the beam size with orbit feedback, while it can be as much as $30 \%$ of the beam size without feedback.

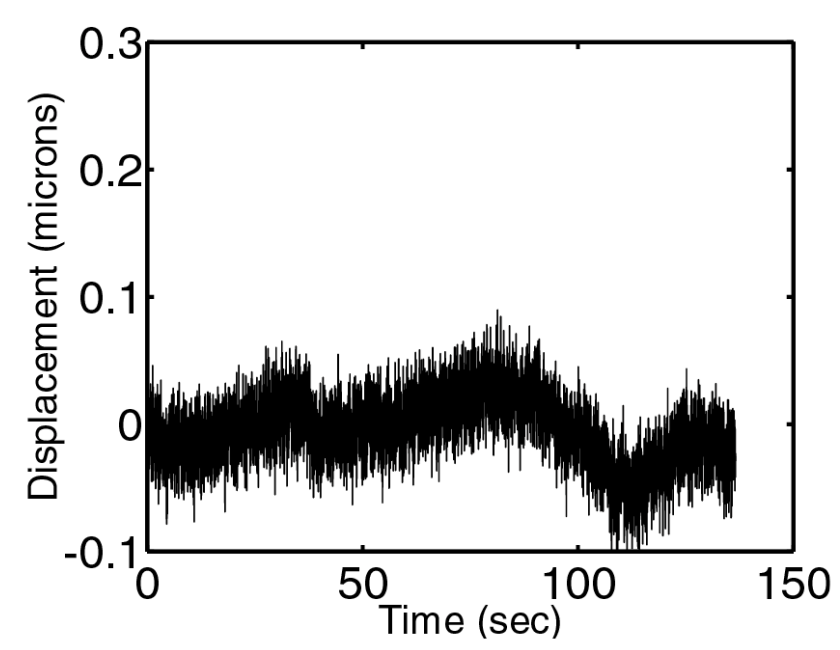

Figure 4: Relative beam motion with respect to the end of the linac with feedback orbit correction. For comparison, the beam size at the end of the linac is $\sigma=1.3$ micron.

At much larger time scales (hours) the main form of ground motion is diffusive ATL motion. The accumulated misalignments of the beamline can be large enough for the beam orbit to deviate significantly from the ideal line after several hours (see Fig.5). The beam emittance degrades if no feedback orbit correction is applied (see Fig.6).

Once the feedback orbit correction is applied, the orbit deviation and the emittance growth become much smaller. In a simulation assuming an initially perfect NLC linac (Fig.6), the emittance growth remains below 10\% for almost two hours. For these linac and ground motion parameters, a beam-based alignment procedure to restore the smoothness of the beamline would need to be applied every few hours. 


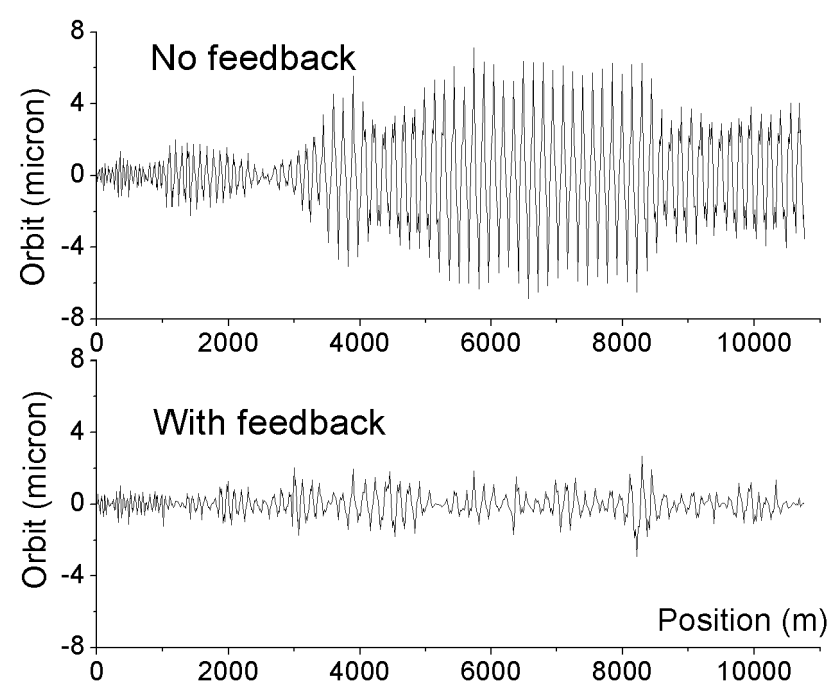

Figure 5: Beam orbit in the linac after 30 minutes with slow ground motion without and with orbit feedback.

\section{FUTURE WORK}

The performance of the NLC linac feedback orbit correction as shown by the results given above should not be considered the ultimate performance of the system. We believe that further improvements are possible. In particular, it would be desirable to reduce the amplification of high frequency motion Nonetheless, the simulated performance is adequate to provide stable beam quality for the NLC. Additional work is needed to optimize feedback performance in the presence of additional imperfections such as changes in klystron phase or energy profile. The feedback configuration planned for NLC depends upon a knowledge of the beam transport matrices over very long distances. If the machine model is not sufficiently accurate, the matrices may also be measured (calibration) or calculated adaptively. Simulations are needed to evaluate the effectiveness of these techniques. Another issue is the interaction between the feedback systems and other tuning procedures such as beam-based alignment, which will move the linac elements during normal operation. Long timeline simulations of a full linac including ground motion, feedback and alignment are planned.

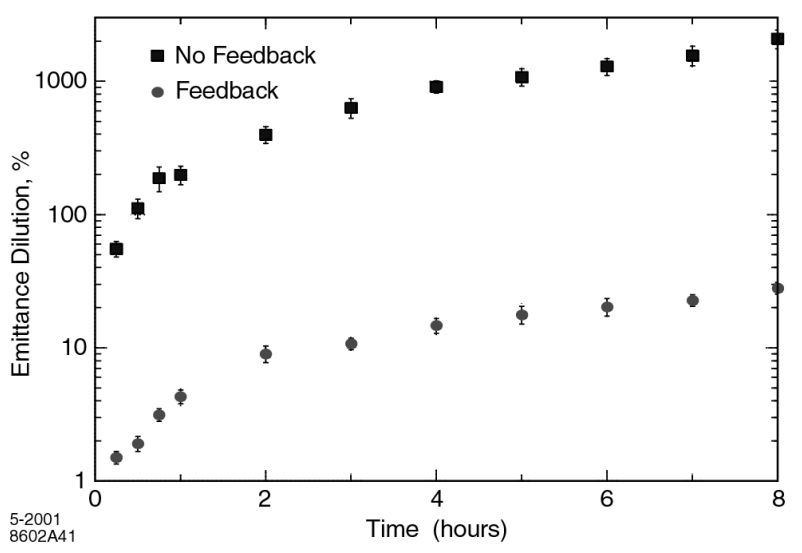

Figure 6: Simulation of the NLC main linac for $500 \mathrm{GeV}$ final energy and an ATL coefficient of $5 \times 10^{-7} \mu \mathrm{m}^{2} / \mathrm{m} / \mathrm{s}$; with and without feedback orbit correction.

\section{CONCLUSION}

We have investigated the performance of the NLC linac orbit correction feedback systems in the presence of ground motion. Simulations indicate that the feedback can adequately stabilize the beam quality and provide long term stability as well as ease and efficiency of operation.

\section{REFERENCES}

[1] NLC ZDR Design Group, SLAC Report-474 (1996).

[2] B. Baklakov, et al. Tech. Phys. 38, 894 (1993).

[3] V. Shiltsev, in Proc. IWAA95, KEK-95-12, 1995.

[4] A.Seryi, T.Raubenheimer, SLAC-PUB-8595, 2000.

[5] A.Seryi, in Proc. of Linac 96.

[6] R. Assmann, et al., SLAC-PUB-7577, 1997.

[7] T. Himel, et al., PAC93; SLAC-PUB-6125 (1993).

[8] L.Hendrickson, et al., ICALEPCS; SLAC-PUB-5683 1991.

[9] L.Hendrickson, et al., LINAC2000; SLAC-PUB$8585,2000$. 